\title{
\begin{tabular}{l|l} 
Mitraries & DSpace@MIT
\end{tabular}
}

\author{
MIT Open Access Articles
}

\section{Fundamental limits of super-resolution microscopy by dielectric microspheres and microfibers}

The MIT Faculty has made this article openly available. Please share how this access benefits you. Your story matters.

Citation: Astratov, V. N., et al. “Fundamental Limits of Super-Resolution Microscopy by Dielectric Microspheres and Microfibers." Edited by Alexander N. Cartwright, Dan V. Nicolau, and Dror Fixler. Nanoscale Imaging, Sensing, and Actuation for Biomedical Applications XIII, February 2016, San Francisco, California, USA, SPIE, April 2016 (c) 2016 SPIE

As Published: http://dx.doi.org/10.1117/12.2212762

Publisher: SPIE

Persistent URL: http://hdl.handle.net/1721.1/115076

Version: Final published version: final published article, as it appeared in a journal, conference proceedings, or other formally published context

Terms of Use: Article is made available in accordance with the publisher's policy and may be subject to US copyright law. Please refer to the publisher's site for terms of use. 


\section{Fundamental limits of super- resolution microscopy by dielectric microspheres and microfibers}

V. N. Astratov, A. V. Maslov, K. W. Allen, N. Farahi, Y. Li, et al.

V. N. Astratov, A. V. Maslov, K. W. Allen, N. Farahi, Y. Li, A. Brettin, N. I. Limberopoulos, D. E. Walker, A. M. Urbas, V. Liberman, M. Rothschild, "Fundamental limits of super-resolution microscopy by dielectric microspheres and microfibers," Proc. SPIE 9721, Nanoscale Imaging, Sensing, and Actuation for Biomedical Applications XIII, 97210K (22 April 2016); doi: $10.1117 / 12.2212762$

SPIE. Event: SPIE BiOS, 2016, San Francisco, California, United States 


\title{
Fundamental limits of super-resolution microscopy by dielectric microspheres and microfibers
}

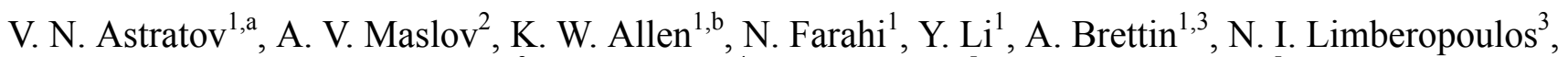 \\ D. E. Walker Jr. ${ }^{3}$, A. M. Urbas ${ }^{4}$, V. Liberman, ${ }^{5}$ and M. Rothschild ${ }^{5}$ \\ ${ }^{1}$ Department of Physics and Optical Science, Center for Optoelectronics and Optical Communication, \\ University of North Carolina at Charlotte, Charlotte, NC 28223-0001, USA \\ ${ }^{2}$ Department of Radiophysics, University of Nizhny Novgorod, Nizhny Novgorod, Russia \\ ${ }^{3}$ Air Force Research Laboratory, Sensors Directorate, Wright-Patterson AFB, OH 45433 USA \\ ${ }^{4}$ Air Force Research Laboratory, Materials and Manufacturing Directorate, Wright Patterson AFB, \\ OH 45433 USA \\ ${ }^{5}$ Lincoln Laboratory, Massachusetts Institute of Technology, Boston, Massachusetts, USA
}

\begin{abstract}
In recent years, optical super-resolution by microspheres and microfibers emerged as a new paradigm in nanoscale label-free and fluorescence imaging. However, the mechanisms of such imaging are still not completely understood and the resolution values are debated. In this work, the fundamental limits of super-resolution imaging by high-index barium-titanate microspheres and silica microfibers are studied using nanoplasmonic arrays made from Au and Al. A rigorous resolution analysis is developed based on the object's convolution with the point-spread function that has width well below the conventional $(\sim \lambda / 2)$ diffraction limit, where $\lambda$ is the illumination wavelength. A resolution of $\sim \lambda / 6-\lambda / 7$ is demonstrated for imaging nanoplasmonic arrays by microspheres. Similar resolution was demonstrated for microfibers in the direction perpendicular to the fiber axis with hundreds of times larger field-of-view in comparison to microspheres. Using numerical solution of Maxwell's equations, it is shown that extraordinary close point objects can be resolved in the far field, if they oscillate out of phase. Possible super-resolution using resonant excitation of whispering gallery modes is also studied.
\end{abstract}

Keywords: Optical super-resolution; near-field microscopy; confocal microscopy

\section{INTRODUCTION}

Optical microscopy is one of the most important and widely spread experimental techniques which can be found in practically any biomedical or microphotonics laboratory. However, the resolution of standard microscopes cannot exceed the diffraction limit which forms an unavoidable barrier for any far-field imaging system. More specific Abbe, Rayleigh, Sparrow, and Houston resolution criteria show that the limit of lateral resolution is close to a half of the wavelength $(\lambda)$. Increasing the resolution beyond the diffraction limit requires new physical principles such as detection of the optical near-fields or use of strong non-linear effects. ${ }^{1}$ In recent years, fluorescence (FL) microscopy achieved the resolution beyond the diffraction limit due to nanoscale dimensions of light sources (dye molecules) combined with the nonlinear optical effects. ${ }^{1}$ However it also has many drawbacks such as photobleaching and, most importantly, staining the biological samples with dyes is not always a desirable option. ${ }^{2}$

The label-free microscopy (LFM) relies on such effects as transient absorption ${ }^{3}$, super-oscillation ${ }^{4}$, and hyperlens imaging ${ }^{5}$. However, LFM is developing more slowly than FL microscopy for two reasons. First, the LFM mechanisms rely on subtler light-scattering processes in nanoscale objects that result in lower effective image contrasts. Second, the experimental quantification of resolution is complicated in LFM due to the lack of good "point"-sources.

\footnotetext{
a'Email: astratov@uncc.edu

${ }^{b}$ Present address: Advanced Concepts Laboratory, Georgia Tech Research Institute, Atlanta, GA 30318-5712, USA
} 
Nanoscopy by microspheres emerged a surprisingly simple imaging technique ${ }^{6,7}$ where a dielectric microsphere is placed in contact with the investigated object and its virtual image is observed using a conventional microscope, as shown in Figs. 1(a-c). It permits FL and LFM imaging. An important advancement in this area was a proposal of using high-index $(n \sim 2)$ microspheres immersed in liquids or embedded in slabs. ${ }^{8,9}$ It makes possible the application of this technology for imaging biomedical objects. ${ }^{10,11}$ Furthermore, imaging by high-index, liquid-immersed spheres resulted in higher-quality and better resolution images of nanoplasmonic structures ${ }^{9,12-14}$.

In this work, we study the fundamental resolution limits of microsphere-assisted imaging both experimentally and using numerical modeling. We use rigorous resolution criteria based on convolution with the point spread function (PSF) and show that resolutions $\sim \lambda / 6-\lambda / 7$ can be realized for imaging nanoplasmonic arrays by microspheres. Our numerical modeling shows that better than diffraction-limited resolution can be realized under conditions of resonant excitation of whispering gallery modes (WGMs) in microspheres; however the resolution advantage is rather limited and typically does not exceed 1.5. On the other hand, we show that extraordinarily small features can be resolved in the images of objects emitting light coherently.

\section{METHOD OF EXPERIMENTAL QUANTIFICATION OF RESOLUTION}

To quantify the resolution in LFM methods, researchers often use larger-scale arrays containing objects with recognizable shape such as periodic stripes, stars, holes, dimers or clusters. ${ }^{4,6,9,14} \mathrm{An}$ idea of this approach is that the resolution can be estimated based on the minimal feature sizes which can be discerned in the optical images. For closely-spaced metallic cylinders (or holes) an edge-to-edge gap ( $g$ ) is usually accepted as a resolution measure, see Fig. 1(d). We showed, however, that this approach can lead to overestimated resolution values. We developed more rigorous quantification of super-resolution based on a convolution of the arbitrarily shaped objects with the two-dimensional (2-D) point spread function (PSF) ${ }^{15-18}$ The proposed method can be viewed as an integral form of the super-resolution quantification accepted in FL microscopy where the PSF width can be narrower than the diffraction limit. As shown Figs. 1(e,f), we demonstrated imaging of metallic dimers and bowties with $\sim \lambda / 7$ resolution, well in excess of the diffraction limit.
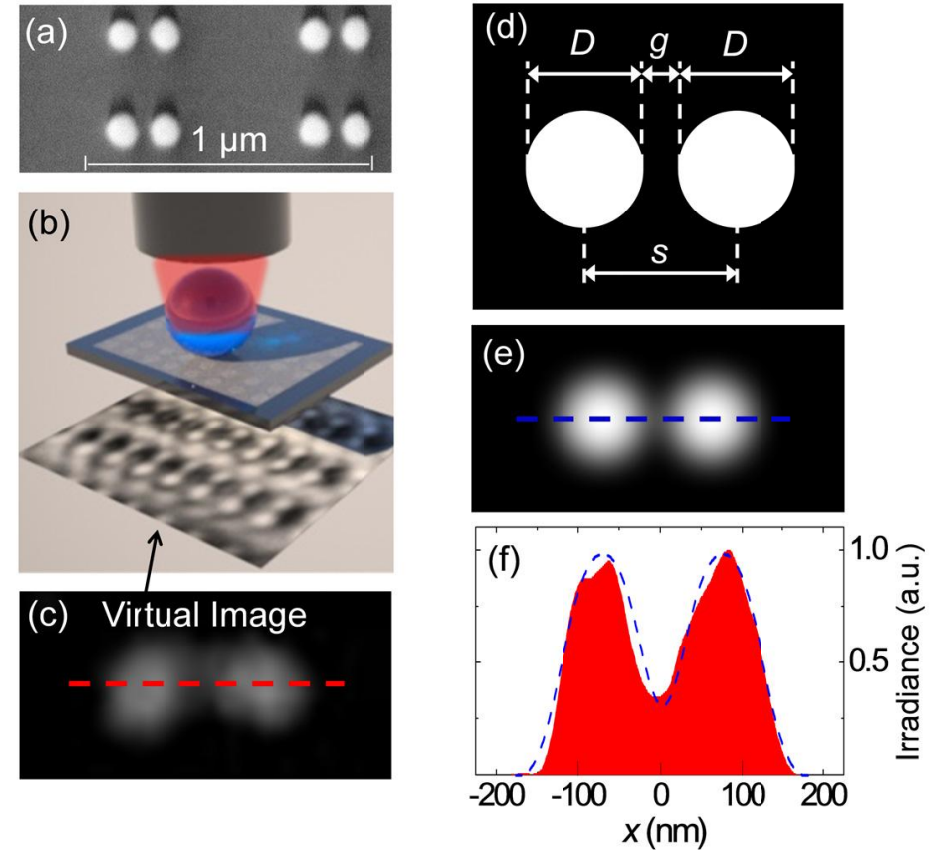

Figure 1. (a) Array of Au dimers with $D=110 \mathrm{~nm}$ and $g=40 \mathrm{~nm}$, (b) microscope setup, (c) virtual image, (d) drawn two-circle object, (e) convoluted image with the PSF width $\sim \lambda / 7$, and (f) comparison of calculated (blue dashed lines) and measured at $\lambda=405 \mathrm{~nm}$ (red profile) image irradiance profile. 


\section{EXPERIMENTAL RESULTS}

To develop a novel optical component for enhancing the microscope's resolution, we fabricated polydimethylsiloxane (PDMS) slabs with embedded high-index $(n \sim 2) \mathrm{BaTiO}_{3}$ microspheres and showed for the first time that these slabs provide the optical super-resolution imaging combined with the surface scanning capability. ${ }^{15-17}$ As illustrated in Fig. 2(a), the PDMS slabs adhere to the surface of nanoplasmonic structures which results in imaging of Au dimers and bowties with $\sim \lambda / 6-\lambda / 7$ resolution. It is shown that the PDMS slabs can be translated along the surface of investigated samples after liquid lubrication. Initially, the resolution is diffraction limited; however the super-resolution gradually recovers as the lubricant evaporates. As shown in Fig. 2(b), the microfiber is another contact lens which provides magnification and super-resolution perpendicular to its axis. The resolution was quantified based on the convolution of arbitrarily shaped objects with the PSF of cylindrical lens. ${ }^{18}$ The resolution $\sim \lambda / 6$ is demonstrated in the direction perpendicular to the fiber with hundreds of times larger field-of-view in comparison to microspheres. We also demonstrated that the microsphere-assisted imaging can be extended into the deep-UV range. ${ }^{19}$
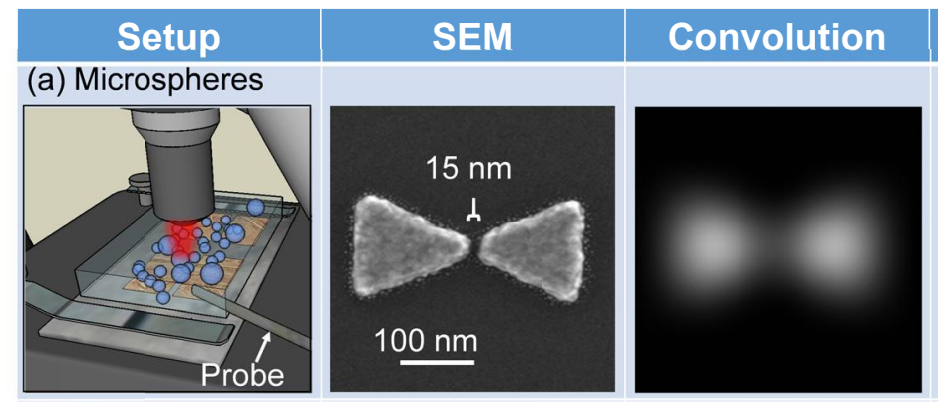

\section{Experiment}

(b) Microfiber
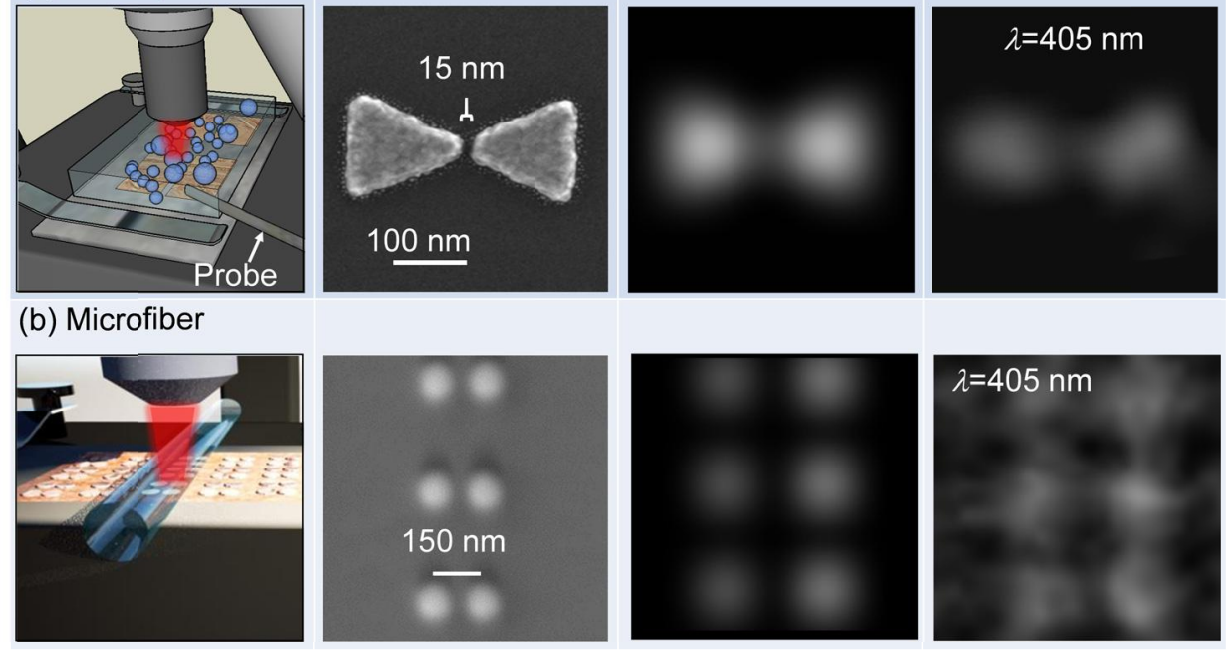

Figure 2. (a) Experimental setup, SEM image of the object, convoluted and experimental images obtained through $4.5 \mu \mathrm{m}$ $\mathrm{BaTiO}_{3}$ microsphere. The sphere is embedded in PDMS slab manipulated by the probe. (b) Same in the case of imaging through $\mathrm{SiO}_{2}$ microfiber with the $12 \mu \mathrm{m}$ diameter without PDMS slab.

\section{NUMERICAL MODELING RESULTS}

Simplicity, massive impact on microscopy, and almost elusive origin of super-resolution by microspheres attracted a significant attention to its theoretical explanation. Initially, it was related to sharper than usual focusing of light by mesoscale spheres. ${ }^{6}$ However, the width of photonic jets is slightly less than $\lambda / 2$ only in small microspheres with diameters about several $\lambda .^{20}$ The enhancement of focusing, therefore, seems to be insufficient to explain the super-resolution which is typically observed in larger spheres.

To get an insight into the imaging mechanisms we performed modeling based on a simplified 2-D model which catches essential physics of real 3-D experimental geometry. The basic idea is illustrated in Fig. 3 using two point sources spaced by $s=\lambda / 2.86$. The dipoles were located at small distance $d=\lambda / 6.28$ below the cylinder with $n_{\mathrm{c}}=1.4$ and $6.4 \lambda$ diameter. The virtual images calculated in a range of focusing depths are seen significantly below the actual location of the two-point object. The incoherent case illustrated in Fig. 3(b) represents a situation which is close to resolving two point sources; however they are not resolved since the virtual image at the depth around 5-6 $\lambda$ has a single maximum. In contrast, in the out-of-phase case the object looks completely "resolved", as shown in Fig. 3(c); however it is merely an interference 
effect. It is interesting, however, that similar coherent effects can play some role in experiments performed with coupled particles with a finite size. Plasmonic structures such as dimers or bowties support coherently coupled modes. The antisymmetric modes would have some properties of two-point dipoles emitting out-of-phase. Recently, we suggested that such modes can be modeled as sinusoidal current distributions which emit light as distributed antennas. ${ }^{21}$

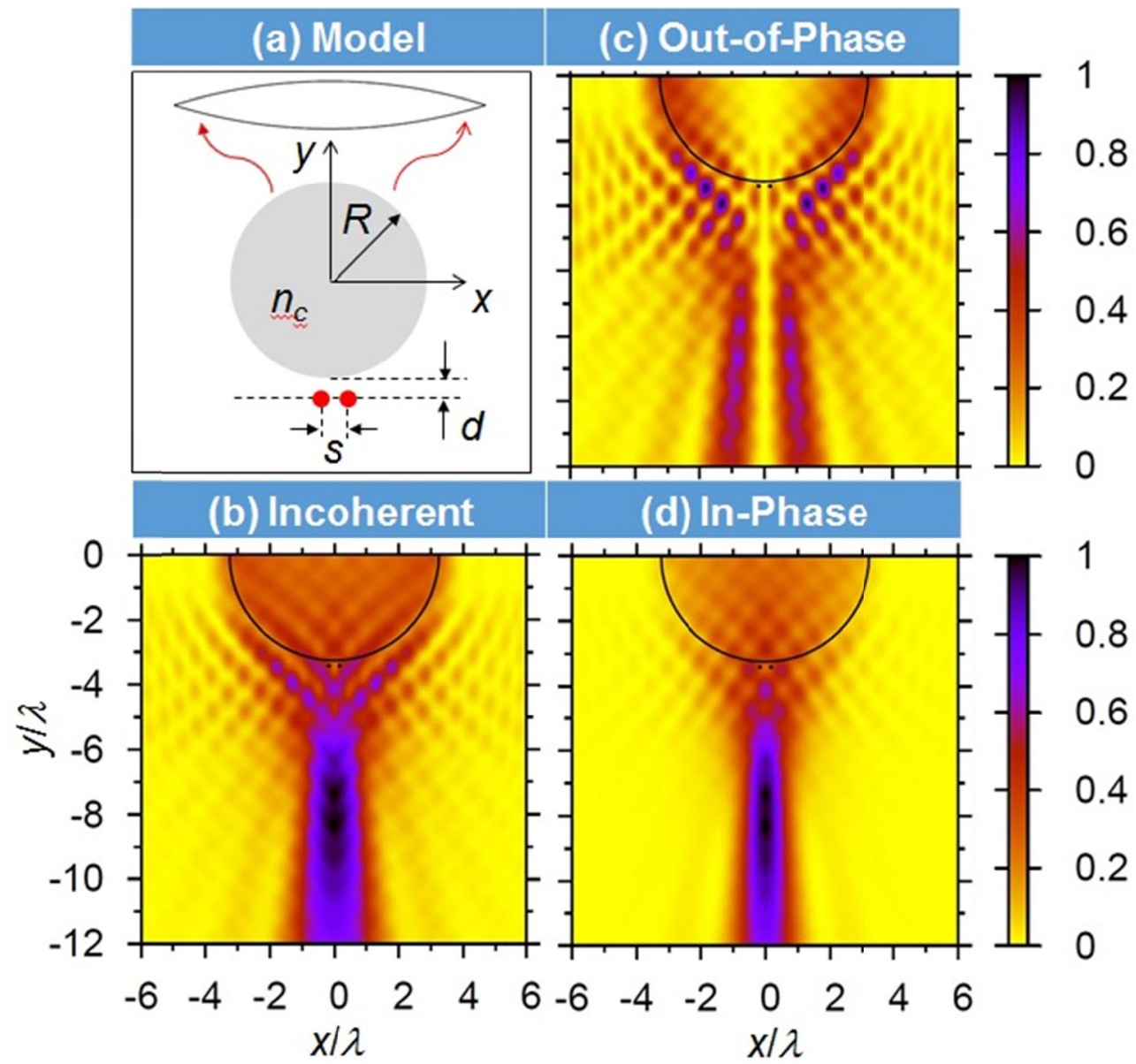

Figure 3. (a) 2-D model of imaging of two-point object with spacing $s=\lambda / 2.86$ located at a depth $d=\lambda / 6.28$ below the cylinder with $n_{\mathrm{c}}=1.4$ and $D=6.4 \lambda$.(b-d) Virtual image intensities calculated as a function of the focusing depth (y) in the incoherent, out-of-phase, and in-phase cases, respectively.

The results in Fig. 3 are obtained out of resonance with WGMs in microspheres. Such resonances are widely used in sensor applications ${ }^{22,23}$ and optical propulsion ${ }^{24-26}$ experiments. Recently, it was demonstrated that the resonant excitation of WGMs can slightly improve the resolution. ${ }^{27}$ We compared resonant and nonresonant imaging cases in Fig. 4 and came to analogous conclusions. It should be noted that the resonant imaging takes place at extremely shallow depth about $1.5 \lambda$ below the cylinder. This imaging takes place with a strong contribution of WGM's optical near-fields and it requires further studies. It is seen, however, that the image in Fig. 4(a) looks "resolved". The standard nonresonant virtual imaging illustrated in Fig. 4(c) takes place at much larger depths about $5 \lambda$ below the sphere. Due to the large extent of the virtual image in vertical direction, this case is much easier to realize and study experimentally, however, incoherent imaging illustrated in Fig. 4(c) shows that the corresponding image is not resolved. 

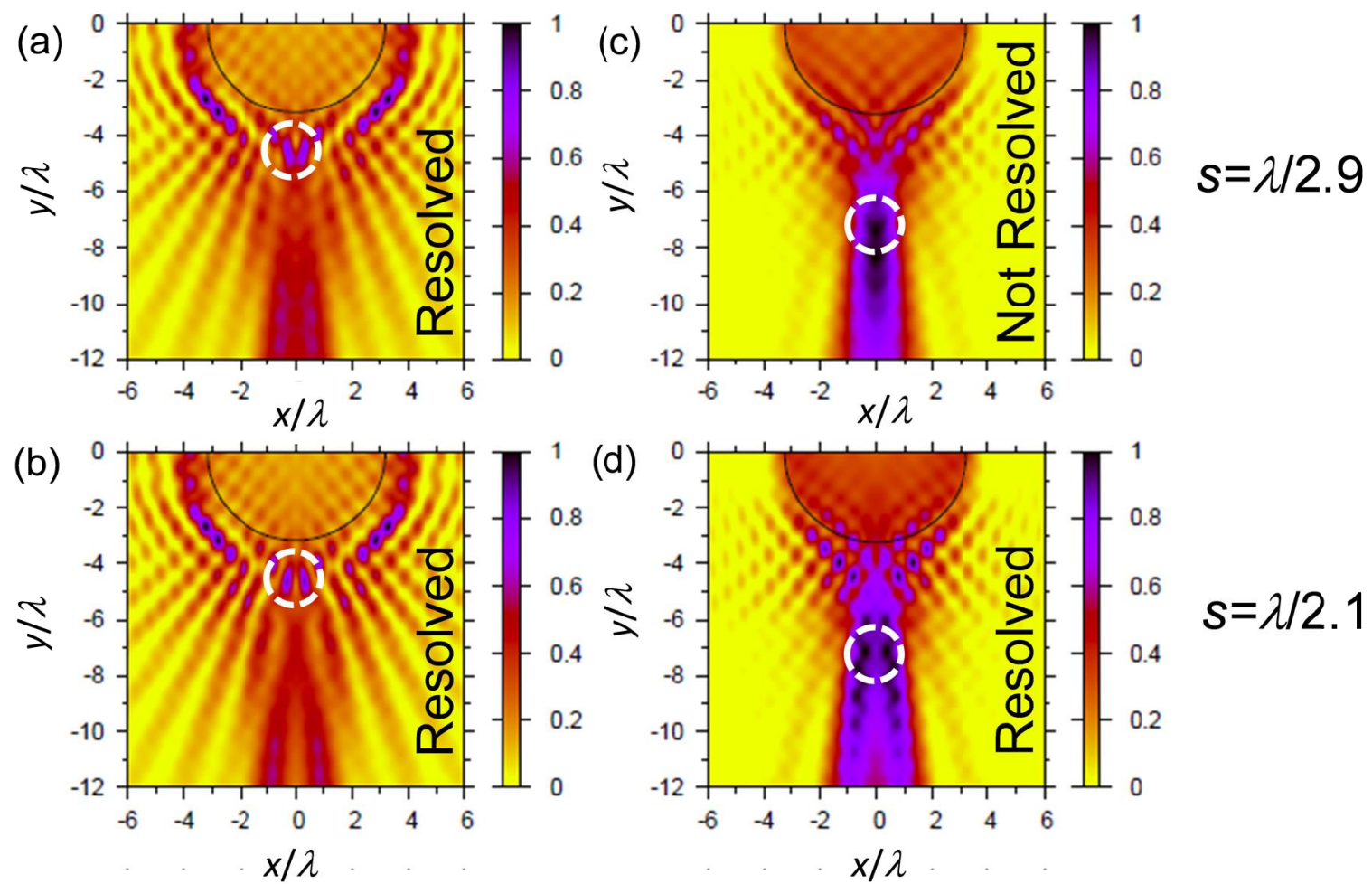

$s=\lambda / 2.1$

Figure 4. Comparison of resonant imaging at the WGM frequency $(a, b)$ with nonresonant imaging $(c, d)$. The calculations are performed for the cases $s=\lambda / 2.9(\mathrm{a}, \mathrm{c})$ and $s=\lambda / 2.1(\mathrm{~b}, \mathrm{~d})$.

In this work we studied the fundamental limits of super-resolution microscopy by microspheres and came to conclusions that there are two drastically different situations when better than diffraction-limited resolution can be realized. The first case is represented by coherent imaging performed at the frequencies where different parts of the coupled nanoscale structure oscillates out-of-phase. This situation is not a violation of the classical diffraction limit since this imaged object has coherent properties and to some extent a prior information about the object is used (antisymmetric mode distribution). In addition, the image does not accurately represent the shape of the object, but it allows a precise localization of some features of the object. The second case is represented by imaging performed at WGM frequencies. This case involves resonant enhancement of the optical near-fields in the vicinity of sphere. It requires more studies, but the likely reason for super-resolution in this case is connected with the image magnification effect produced by the resonantly enhanced optical near fields. Similar effects can play some part in quantification of resolution in recent experimental studies. We plan to study these cases in our future experimental work.

\section{ACKNOWLEDGMENTS}

The work at UNCC was supported by the U.S. Army Research Office through Dr. J. T. Prater under Contract No. W911NF-09-1-0450. The work at UNN was supported in part by the Ministry of Education and Science of the Russian Federation through Agreement No. 02.B.49.21.0003. The UNCC team is also grateful for a support by the Center for Metamaterials, an NSF I/U CRC, award number 1068050, and by Air Force Research Laboratory (AFRL/RYD, AFRL/RXC) through the AMMTIAC contract with Alion Science and Technology and the MCF II contract with UES, Inc. The Lincoln Laboratory portion of this work was supported by the Assistant Secretary of Defense for Research \& Engineering under Air Force Contract \# FA8721-05-C-002. Opinions, interpretations, conclusions and recommendations are those of the author and are not necessarily endorsed by the United States Government. 


\section{REFERENCES}

[1] Hell, S. W., "Nanoscopy with focused light," Ann. Phys. (Berlin) 527, 423-445 (2015).

[2] Weisenburger, S. and Sandoghdar, V., "Light microscopy: an ongoing contemporary revolution," Contemporary Physics 56(2), 123-143 (2015).

[3] Wang, P., Slipchenko, M. N., Mitchell, J., Yang, C., Potma, E. O., Xu, X. and Cheng, J.-X., "Far-field imaging of non-fluorescent species with subdiffraction resolution," Nature Photon. 7(6), 449-453 (2013).

[4] Rogers, E. T. F., Lindberg, J., Roy, T., Savo, S., Chad, J. E., Dennis, M. R. and Zheludev, N. I., “A super-oscillatory lens optical microscope for subwavelength imaging," Nature Mater. 11(5), $432-435$ (2012).

[5] Jacob, Z., Alekseyev, L. V. and Narimanov, E., "Optical Hyperlens: Far-field imaging beyond the diffraction limit," Opt. Express 14(18), 8247-8256 (2006).

[6] Wang, Z., Guo, W., Li, L., Luk'yanchuk, B., Khan, A., Liu, Z., Chen, Z. and Hong, M., “Optical virtual imaging at 50 nm lateral resolution with a white-light nanoscope," Nat. Commun. 2, 218 (2011).

[7] Astratov, V. N, Allen, K. W., Farahi, N., Li, Y., Limberopoulos, N. I., Walker Jr., D. E., Urbas, A. M., Liberman, V. and Rothschild, M., "Optical nanoscopy with contact microlenses overcomes the diffraction limit," SPIE Newsroom, February 1 (2016).

[8] Astratov, V. N. and Darafsheh, A., "Methods and systems for super-resolution optical imaging using high-index of refraction microspheres and microcylinders," US patent 20140355108, priority June 7 (2012).

[9] Darafsheh, A., Walsh, G. F., Dal Negro, L. and Astratov, V. N., "Optical super-resolution by high-index liquid-immersed microspheres," Appl. Phys. Lett. 101(14), 141128 (2012).

[10] Li, L., Guo, W., Yan, Y., Lee, S. and Wang, T., "Label-free super-resolution imaging of adenoviruses by submerged microsphere optical nanoscopy," Light: Science \& Applications 2, e104 (2013).

[11] Yang, H., Moullan, N., Auwerx, J. and Gijs, M. A. M., "Super-resolution biological microscopy using virtual imaging by a microsphere nanoscope," Small 10(9), 1712-1718 (2014).

[12] Krivitsky, L. A., Wang, J. J., Wang, Z. and Luk'yanchuk, B., "Locomotion of microspheres for super-resolution imaging," Sci. Reports 3, 3501 (2013).

[13] Darafsheh, A., Limberopoulos, N. I., Derov, J. S., Walker, Jr., D. E. and Astratov, V. N., "Advantages of microsphere-assisted super-resolution imaging technique over solid immersion lens and confocal microscopies," Appl. Phys. Lett. 104(6), 061117 (2014).

[14] Yan, Y., Li, L., Feng, C., Guo, W., Lee, S. and Hong, M., "Microsphere-coupled scanning laser confocal nanoscope for sub-diffraction-limited imaging at $25 \mathrm{~nm}$ lateral resolution in the visible spectrum," ACS Nano 8(2), 1809-1816 (2014).

[15] Allen, K. W., Farahi, N., Li, Y., Limberopoulos, N. I., Walker Jr., D. E., Urbas, A. M. and Astratov V. N., "Super-resolution imaging by arrays of high-index spheres embedded in transparent matrices," IEEE Proc. of Aerospace and Electronics Conference (NAECON), Dayton, pp. 50-52, June 24-27 (2014).

[16] Allen, K. W., "Waveguide, photodetector, and imaging applications of microspherical photonics," Ph.D. dissertation (University of North Carolina at Charlotte), Chapter 4: Super-Resolution Imaging through Arrays of High-Index Spheres Embedded in Transparent Matrices, pp. 98-122, October (2014).

[17] Allen, K. W., Farahi, N., Li, Y., Limberopoulos, N. I., Walker, Jr., D. E., Urbas, A. M., Liberman, V. and Astratov, V. N., "Super-resolution microscopy by movable thin-films with embedded microspheres: Resolution analysis," Ann. Phys. (Berlin) 527, 513-522 (2015).

[18] Allen, K. W., Farahi, N., Li, Y., Limberopoulos, N. I., Walker Jr., D. E., Urbas, A. M. and Astratov, V. N., "Overcoming the diffraction limit of imaging nanoplasmonic arrays by microspheres and microfibers," Opt. Express 23(19), 24484-24496 (2015).

[19] Allen, K. W., Liberman, V., Rothschild, M., Limberopoulos, N. I., Walker Jr., D. E., Urbas, A. M., and Astratov, V. N., "Deep-UV microsphere-assisted ultramicroscopy," in IEEE Proc. of 17th International Conference on Transparent Optical Networks-ICTON, Paper No. We.P.26 (2015).

[20] Li, X., Chen, Z., Taflove, A. and Backman, V., "Optical analysis of nanoparticles via enhanced backscattering facilitated by 3-D photonic nanojets," Opt. Express 13(2), 526-533 (2005).

[21] Maslov, A. V. and Astratov, V. N., "Imaging of sub-wavelength structures radiating coherently near microspheres," Appl. Phys. Lett. 108(5), 051104 (2016).

[22] Hiremath, K. R. and Astratov, V. N., "Perturbations of whispering gallery modes by nanoparticles embedded in microcavities," Opt. Express 16(8), 5421-5426 (2008). 
[23] Svitelskiy, O., Li, Y., Darafsheh, A., Sumetsky, M., Carnegie, D., Rafailov, E. and Astratov, V. N., "Fiber coupling to $\mathrm{BaTiO}_{3}$ glass microspheres in an aqueous environment,” Opt. Lett. 36(15), 2862-2865 (2011).

[24] Li, Y., Svitelskiy, O. V., Maslov, A. V., Carnegie, D., Rafailov, E. and Astratov, V. N., "Giant resonant light forces in microspherical photonics," Light: Science \& Applications 2, e64 (2013).

[25] Maslov, A. V. and Astratov, V. N., "Microspherical photonics: sorting resonant photonics atoms by using light," Appl. Phys. Lett. 105(12), 121113 (2014).

[26] Li, Y., Maslov, A. V., Limberopoulos, N. I., Urbas, A. M. and Astratov, V. N., "Spectrally resolved resonant propulsion of dielectric microspheres," Laser \& Photonics Rev. 9(2), 263-273 (2015).

[27] Hoang, T. X., Duan, Y., Chen, X. and Barbastathis, G., "Focusing and imaging in microsphere-based microscopy," Opt. Express 23(9), 12337-12353 (2015). 